\title{
Ginkgo biloba delays light-induced photoreceptor degeneration through antioxidant and antiapoptotic properties
}

\author{
MARYAM CHUDHARY ${ }^{1 *}$, CHENGHONG ZHANG ${ }^{2 *}$, SHIYU SONG $^{1}$, XIANG REN ${ }^{1}$ and LI KONG \\ ${ }^{1}$ Department of Histology and Embryology; ${ }^{2}$ Teaching Laboratory of Morphology, \\ College of Basic Medicine, Dalian Medical University, Dalian, Liaoning 116044, P.R. China
}

Received August 24, 2020; Accepted February 5, 2021

DOI: $10.3892 /$ etm.2021.10008

\begin{abstract}
Intense exposure to artificial bright light increases the risk of retinal damage resulting in blurred vision and blindness. Long-term exposure to bright light elevates oxidative stress-induced apoptosis, which results in photoreceptor cell degeneration. However, to the best of our knowledge, the molecular mechanism associated with light-induced retinopathy remains unclear. In the present study, the mechanisms involved in light-induced oxidative stress and apoptosis were investigated along with the protective effects of Ginkgo biloba (EGb 761) in photoreceptor cell degeneration. EGb 761 was administered to mice at a dose of 50 or $100 \mathrm{mg} / \mathrm{kg}$ for 7 days prior to exposure to bright light (5,000 lux for $24 \mathrm{~h}$ ). Furthermore, photoreceptor cell disorders were evaluated using electroretinogram (ERG) and H\&E staining analyses. The expression levels of antioxidant genes and proteins ERK, thioredoxin (Trx) and nuclear factor erythroid 2-related factor 2 (Nrf-2) and the induction of apoptosis cytochrome c (Cyc), cleaved caspase-3 and Bax, were determined by reverse transcription-quantitative PCR and western blotting. ERG and histological analysis revealed that exposure to bright light induced functional and morphological changes to the photoreceptor cells. Exposure to bright light increased the levels of Cyc, cleaved caspase-3 and Bax, and decreased the levels of phosphorylated (p-) Erk, Nrf-2 and thioredoxin (Trx). However, treatment of mice with EGb 761 increased the expression levels of antiapoptotic (Bcl-2) and antioxidant (p-Erk, Trx and Nrf-2)
\end{abstract}

Correspondence to: Dr Li Kong or Dr Xiang Ren, Department of Histology and Embryology, College of Basic Medicine, Dalian Medical University, 9 Lvshun South Road Western Section, Dalian, Liaoning 116044, P.R. China

E-mail: kongli@dmu.edu.cn

E-mail: xiangren@dmu.edu.cn

${ }^{*}$ Contributed equally

Abbreviations: EGb 761, Ginkgo biloba; ONL, outer nuclear layer; ERG, electroretinogram; ROS, reactive oxygen species

Key words: Ginkgo biloba, light damage, retinal degeneration, oxidative stress, apoptosis proteins and decreased the expression levels of the apoptotic genes (Cyc, cleaved caspase- 3 and Bax). Based on these findings, the present study suggested that prolonged exposure to light induces photoreceptor cell degeneration, where EGb 761 treatment may serve a therapeutic effect on the development of photoreceptor cell degeneration.

\section{Introduction}

Light is the ultimate powerful agent for the maintenance of circadian clocks. However, prolonged exposure to artificial light disturbs circadian rhythms $(1,2)$ and leads to retinal degeneration, such as macular degeneration and retinitis pigmentosa, which is associated with photoreceptor degeneration (3). Prolonged exposure to bright light mediates photoreceptor cell death, which is an irrevocable retinal disorder and causes impaired vision function, vision loss or blindness $(4,5)$. However, to the best of our knowledge, the pathogenesis of light-induced damage to the retina is unclear.

Excessive light exposure promotes a cascade of oxidative stress-associated events involved in retinal degeneration $(6,7)$, which serves a pivotal role in accelerating several degenerative diseases such as age-related macular degeneration (AMD) and retinitis pigmentosa (RP) (3). Overproduction of reactive oxygen species (ROS) can lead to retinal functional and morphological changes that result in vision impairment $(8,9)$. Nuclear factor erythroid 2-related factor 2 (Nrf-2) is a redox regulator (10) that belongs to the leucine zipper transcription factor family, which normally binds to keap1 in order to form a complex. In response to oxidative stress, keap1 activity is reduced, which increases Nrf-2 levels to maintain cellular homeostasis (11). Thioredoxin (Trx) is an antioxidant, which contains an active site Cys-Gly-Pro-Cys domain (12). The latter serves an important role in cellular oxidative stress $(13,14)$. Trx is a potent regulator of Nrf-2 that serves an important role in redox signaling (15). Additionally, it regulates the antiapoptotic signaling pathway, which can protect photoreceptor cells from retinal degeneration $(15,16)$.

Ginkgo biloba (EGb-761) is a traditional Chinese medicine, which contains a naturally occurring compound extracted from the green dried leaves of the Ginkgo biloba tree $(17,18)$. EGb-761 has been used to treat chronic inflammatory and autoimmune disease in patients for several decades (18). Due to its antioxidants and cytoprotective properties, EGb-761 
has received considerable attention regarding the prevention of neurodegenerative diseases (19). It can be used to prevent oxidative stress by regulating antioxidant proteins, such as $\mathrm{Nrf-2}$, which is a redox regulator that stabilizes oxidative stress $(20,21)$ by activating the Erk signaling pathway (22). A light damage mouse model has been previously used to explore the protective effect of Ginkgo biloba in the development of chronic diseases (23).

Apoptosis, also referred to as programmed cell death, is the main cause of photoreceptor cell death involved in retinal degeneration (5). Apoptosis occurs via the activation of the Bax-dependent mitochondrial cascade, which releases cytochrome $c$ (Cyc) into the cytoplasm and consequently initiates the activation of cleaved caspase- $3(15,24,25)$. This suppresses $\mathrm{Bcl} 2$, induces apoptosis via the $\mathrm{Bax} / \mathrm{Bcl} 2$ and caspase-mediated signaling pathways, and leads to retinal degeneration $(25,26)$. As discussed in previous studies, pretreatment with EGb-761 decreases cell death via the $\mathrm{Bax} / \mathrm{Bcl} 2$ signaling pathway and exerts a protective effect against apoptosis that delays photoreceptor degeneration $(27,28)$. However, the protective effect of EGb-761 on light-induced photoreceptor degeneration and the associated mechanism of action are not fully understood.

In the present study, a light damage mouse model was used to evaluate the effects of EGb 761 on light-induced photoreceptor degeneration and to explore the associated mechanism of action. The present study aimed to provide evidence for the treatment or prevention of light-induced photoreceptor degeneration.

\section{Materials and methods}

Animal model. In total, 20 male and 20 female BALB/cJ mice (age, 8-12 weeks), weighing 18-20 g (Dalian Medical University Laboratory Animal Center) were housed for 1 week for acclimization in the lab environment. All mice were maintained at $22 \pm 2^{\circ} \mathrm{C}$ and $30-70 \%$ humidity, under $12-\mathrm{h}$ dark/light cycle with free access of food and water supply. All procedures were approved by the Institutional Animal Care and Use Committee of the Dalian Medical University (Dalian, China). The extract of Ginkgo biloba leaves (EGb-761) was provided by Sigma-Aldrich; Merck KGaA.

Drug administration. Mice were randomly divided into four groups as follows: No treatment (Control); Light (L); Light + Low Ginkgo $(\mathrm{L}+\mathrm{LG})$; and Light + High Ginkgo $(\mathrm{L}+\mathrm{HG})$. A total of 10 mice were used in each group. The mice were pretreated with EGb-761 for 7 days and received an intraperitoneal injection once per day, at a dose of $50 \mathrm{mg} / \mathrm{kg}$ $(\mathrm{L}+\mathrm{LG})$ or $100 \mathrm{mg} / \mathrm{kg}(\mathrm{L}+\mathrm{HG})$. The drug was dissolved in saline solution. Mice in the control and light groups received intraperitoneal injections of normal saline solution.

Light exposure. The mice were adapted in the dark for $24 \mathrm{~h}$ prior to the experiments and exposed to 5,000 lux (diffuse horizontal illuminance) of white fluorescent light $(400-750 \mathrm{~nm})$ for $24 \mathrm{~h}$. The procedure of light exposure was the same as that described in a previous study (29). During light exposure, one mouse was maintained per cage with a sufficient supply of food and water. The eyes of four mice were enucleated at
2 weeks after light exposure for outer nuclear layer (ONL) thickness analysis and electroretinography assessment. The eyes of six mice were enucleated immediately after light exposure to detect possible rapid changes by western blotting and reverse transcription-quantitative PCR (RT-qPCR). After electroretinogram (ERG) detection, the mice were sacrificed using an overdose of carbon dioxide (30\% volume/min) at the end of the experiments before enucleation.

$E R G$. Prior to light exposure, the mice were adapted to the dark overnight and anesthetized with an intraperitoneal injection of pentobarbital-sodium $(60 \mathrm{mg} / \mathrm{kg}) 30 \mathrm{~min}$ before the experiment. The pupils were dilated by applying one drop $0.5 \%$ tropicamide prior to performing ERG analysis. ERG was performed using an LKC ERG system (GT-2008v-3; Gotec, Inc.; http://www.gotechina.com/product/?110_471. html). Phototopic ERGs were generated at $3 \mathrm{~cd} . \mathrm{sec} / \mathrm{m}^{2} \mathrm{flash}$ intensity. Each flash lasted for $5 \mathrm{msec}$ and the interval was $2 \mathrm{sec}$. The amplitudes of the a-wave and b-wave were recorded. The ERG wave from both eyes of the same animal was recorded simultaneously.

Histological analysis. H\&E staining was performed for morphological analysis as described in our previous study (30). The eyeball was removed and fixed for $24 \mathrm{~h}$ at room temperature in Bouin's solution (glacial acetic acid: Formaldehyde: Saturated picric acid=1:5:15; freshly prepared). Subsequently, it was placed in $70 \%$ ethanol for $48 \mathrm{~h}$ at room temperature, or until the yellow color of Bouin's solution was faint or disappeared. For dehydration, each eyeball was passed through a series of alcohol solutions as follows: 80 and $90 \%$ ethanol for 15 min each, 95\% ethanol (twice for $10 \mathrm{~min}$ each) and $100 \%$ ethanol (twice for $10 \mathrm{~min}$ each). The eyeballs were subsequently passed through xylene for $10 \mathrm{~min}$ and then $5 \mathrm{~min}$, respectively, before the tissues were soaked in paraffin wax for $10 \mathrm{~min}$ at $58^{\circ} \mathrm{C}$. The paraffin-embedded tissues were sectioned using a thickness margin of $5 \mu \mathrm{m}$. The sections were deparaffinized first using xylene followed by a descending ethanol gradient. The slices were stained with H\&E each for $5 \mathrm{~min}$ at room temperature. The ONL thickness of the retinal optic nerve was measured at eight different positions at a distance of $0.22 \mathrm{~mm}$ from the inferior hemisphere to the superior hemisphere using light microscope with Element BR software (magnification, x40; Ver5.30.00; Nikon Corporation).

$R T-q P C R$. The total RNA was extracted using RNAiso Plus (Takara Bio, Inc.) from the retina tissue according to the manufacturer's protocols. The concentration of RNA was measured using a NanoDrop spectrometer (NanoDrop Technologies; Thermo Fisher Scientific, Inc.). cDNA was synthesized from $1 \mu \mathrm{g}$ total RNA using the PrimeScript ${ }^{\mathrm{TM}}$ RT reagent Kit (Perfect Real Time; Takara Bio, Inc.) according to the manufacturer's protocol. qPCR was performed using TB Green ${ }^{\circledR}$ Premix Ex Taq ${ }^{\mathrm{TM}}$ (Takara Bio, Inc.) as follows: Initial denaturation at $95^{\circ} \mathrm{C}$ for $30 \mathrm{sec}$; followed by 40 cycles of amplification $\left(95^{\circ} \mathrm{C}\right.$ for $30 \mathrm{sec}, 95^{\circ} \mathrm{C}$ for $30 \mathrm{sec}, 55^{\circ} \mathrm{C}$ for $30 \mathrm{sec}$ and $72^{\circ} \mathrm{C}$ for $30 \mathrm{sec}$ ), $95^{\circ} \mathrm{C}$ for $1 \mathrm{~min}, 55^{\circ} \mathrm{C}$ for $30 \mathrm{sec}$, $95^{\circ} \mathrm{C}$ for $30 \mathrm{sec}$. GAPDH was used as an internal control. The $2^{-\Delta \Delta \mathrm{Cq}}$ method (31) was used to analyze the data. The primer sequences used were: GAPDH forward, 5'-TGTGATGGG 

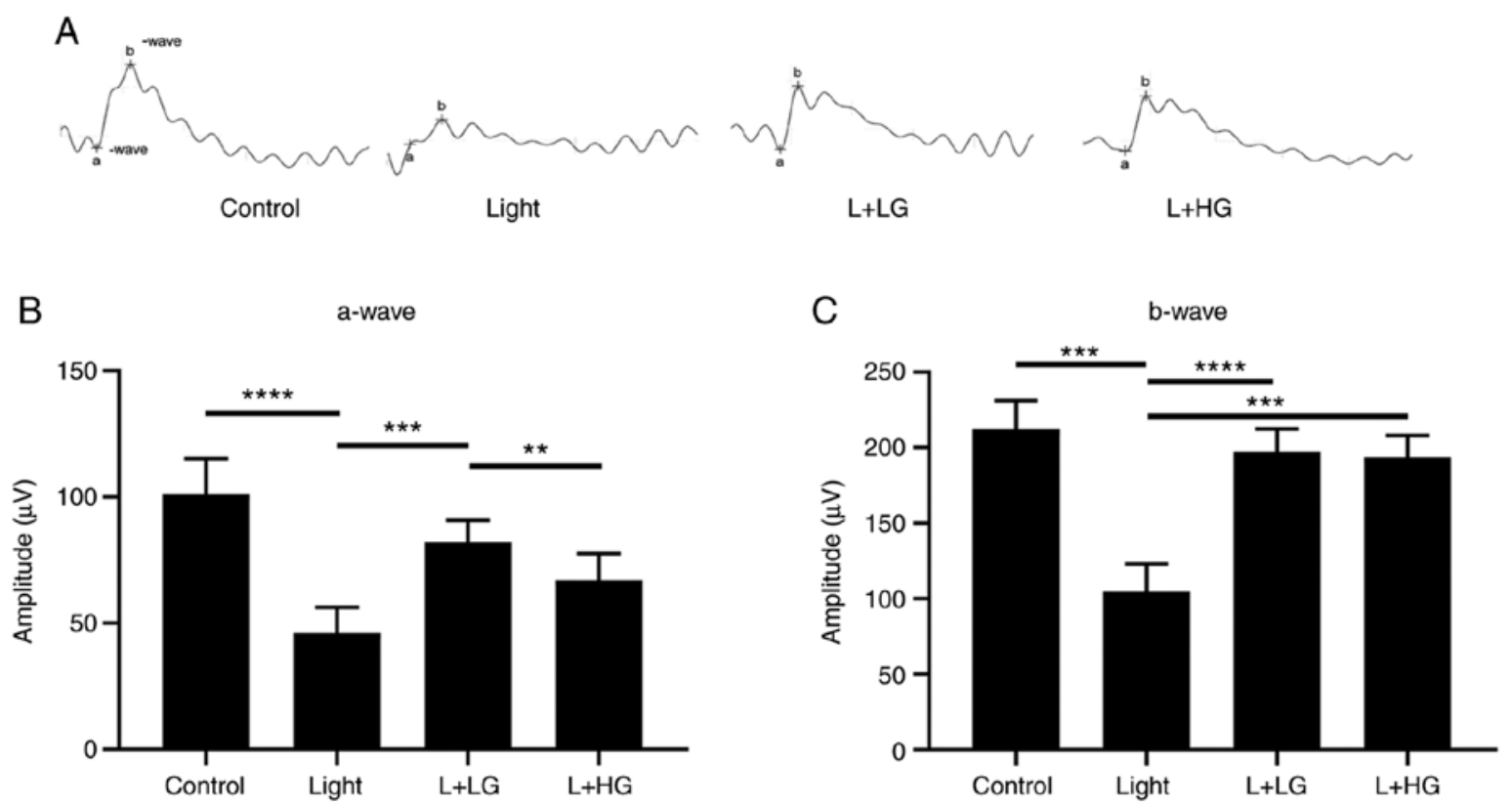

Figure 1. EGb-761 protects the retinal function measured by electroretinogram. (A) Amplitudes of a-wave and b-wave exposed to 5,000 lux of light for $24 \mathrm{~h}$ with/without EGb-761 pre-treatment. Statistical analysis of the (B) a-wave and (C) b-wave. The data are presented as the mean \pm SD ( $n=3$ in each group). ${ }^{* *} \mathrm{P}<0.01,{ }^{* * * *} \mathrm{P}<0.001,{ }^{* * * * *} \mathrm{P}<0.0001$. EGb-761, L + HG (100 mg/kg); L + LG (50 mg/kg). L, light, LG, Low Ginkgo; HG, high Ginkgo.

TGTGAACCACGAGAA-3' and reverse, 5'-GAGCCCTTC CACAATGCCAAAGTT-3'; BCL2 antagonist/killer 1 (Bak-1) forward, 5'-ACGAACTCTTCACCAAGATCGCCT-3' and reverse, 5'-TCAAACCACGCTGGTAGACGTACA-3'; and Cyc forward, 5'-AGATGTTGTTGATGATGGGCCTGC-3' and reverse, 5'-AAGCCAGCTTTCGACTCTTCAGGA-3'.

Western blotting. Total protein was extracted from retinal tissues for 30 min using freshly prepared ice-cold lysis buffer (cat. no. KGP2100; Nanjing Keygen Biotech Co., Ltd.). The lysate was homogenized and centrifuged at $1,000 \mathrm{x} \mathrm{g}$ for $10 \mathrm{~min}$ at $4^{\circ} \mathrm{C}$ before the supernatant was collected and centrifuged again at $12,000 \mathrm{x}$ g for $20 \mathrm{~min}$ at $4^{\circ} \mathrm{C}$. The supernatant was separated and preserved at $-80^{\circ} \mathrm{C}$ for subsequent use. The protein concentration was measured using a bicinchoninic acid protein assay kit (Nanjing KeyGen Biotech Co., Ltd.). Equal amount $(20 \mu \mathrm{g})$ of proteins were separated by SDS-PAGE (12-15\%) and transferred onto polyvinylidene difluoride membranes, which were blocked using 5\% nonfat milk at room temperature for $1 \mathrm{~h}$. Subsequently, the membranes were incubated with primary antibodies against GAPDH (dilution, 1:1,000; ProteinTech Group, Inc.), Bcl2 (dilution, 1:1,000; ABclonal Biotech Co., Ltd.), Bax (dilution, 1:1,000; ABclonal Biotech Co., Ltd.), cleaved caspase-3 (dilution, 1:1,000; Nanjing KeyGen Biotech Co., Ltd.), ERK (dilution, 1:500; Beyotime Institute of Biotechnology), phosphorylated (p-)Erk (dilution, 1:500; Beyotime Institute of Biotechnology), Trx (dilution, 1:200; Santa Cruz Biotechnology, Inc.) and Nrf-2 (dilution, 1:500; cat. no. 163961-1-AP; ProteinTech Group, Inc.) overnight at $4^{\circ} \mathrm{C}$. The membranes were washed with $1 \mathrm{X}$ TBS with $0.1 \%$ Tween 20 (TBST) three times for $15 \mathrm{~min}(5 \mathrm{~min} /$ wash). Subsequently, the membranes were probed with goat anti-rabbit IgG for $1 \mathrm{~h}$ at room temperature, and washed with 1X TBST three times for $45 \mathrm{~min}(15 \mathrm{~min} /$ wash). The protein bands were visualized using enhanced chemiluminescence agent (Beijing Solarbio Science \& Technology Co., Ltd.), and Image lab (version: 4. 1.0.2177; Bio-Rad Laboratoties, Inc.) software was used to analyze the data.

Statistical analysis. The experimental data were obtained from at least three independent experiments. The data are presented as the mean $\pm \mathrm{SD}$. One-way ANOVA was used to analyze the data. All data were analyzed using GraphPad Prism 8 software (GraphPad Software, Inc.). P $<0.05$ was considered to indicate a statistically significant difference.

\section{Results}

Protective effect of EGb-761 against light-induced retinal degeneration. To evaluate the protective effect of EGb-761 on light-induced retinal damage, mice were exposed to 5,000 lux of white light for $24 \mathrm{~h}$ and treated with EGb-761 for 7 days prior to exposure to light. To analyze the visual function of the retina, an ERG was performed (Fig. 1). The results suggested that the amplitudes of the a-wave and b-wave were significantly decreased in mice exposed to 5,000 lux of bright light for $24 \mathrm{~h}$ compared with the levels noted in the control group (Fig. 1). However, a fractional protective effect could be observed when mice were treated with EGb-761 (L + LG and $\mathrm{L}+\mathrm{HG}$ ) for 7 days prior to exposure to white light. As shown in Fig. 1, the amplitude of the a-wave was increased $(\mathrm{L}+\mathrm{LG})$ and the amplitudes of the $b$-wave were significantly increased in the EGb-761 (L + LG and L + HG) groups compared with that in the light-damage group (Fig. 1A-C).

EGb-761 delays light-induced photoreceptor degeneration. To evaluate the protective effect of EGb-761 on photoreceptor degeneration, H\&E staining was performed. The morphological evaluation using $\mathrm{H} \& \mathrm{E}$ staining indicated a massive 

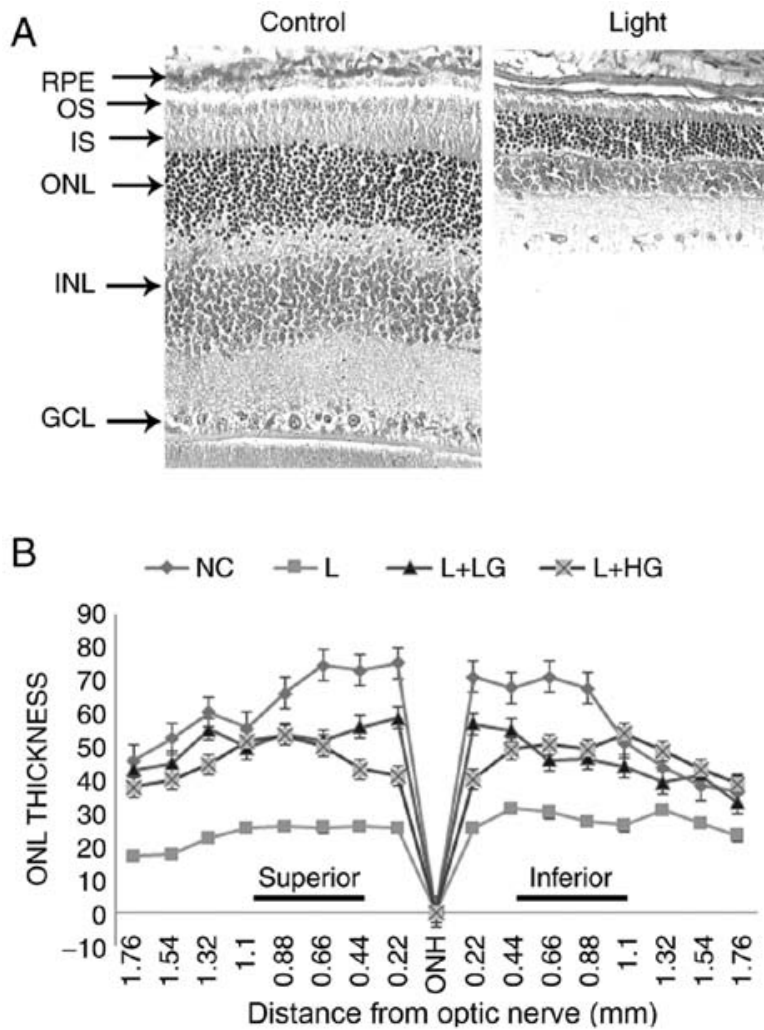

$L+L G$
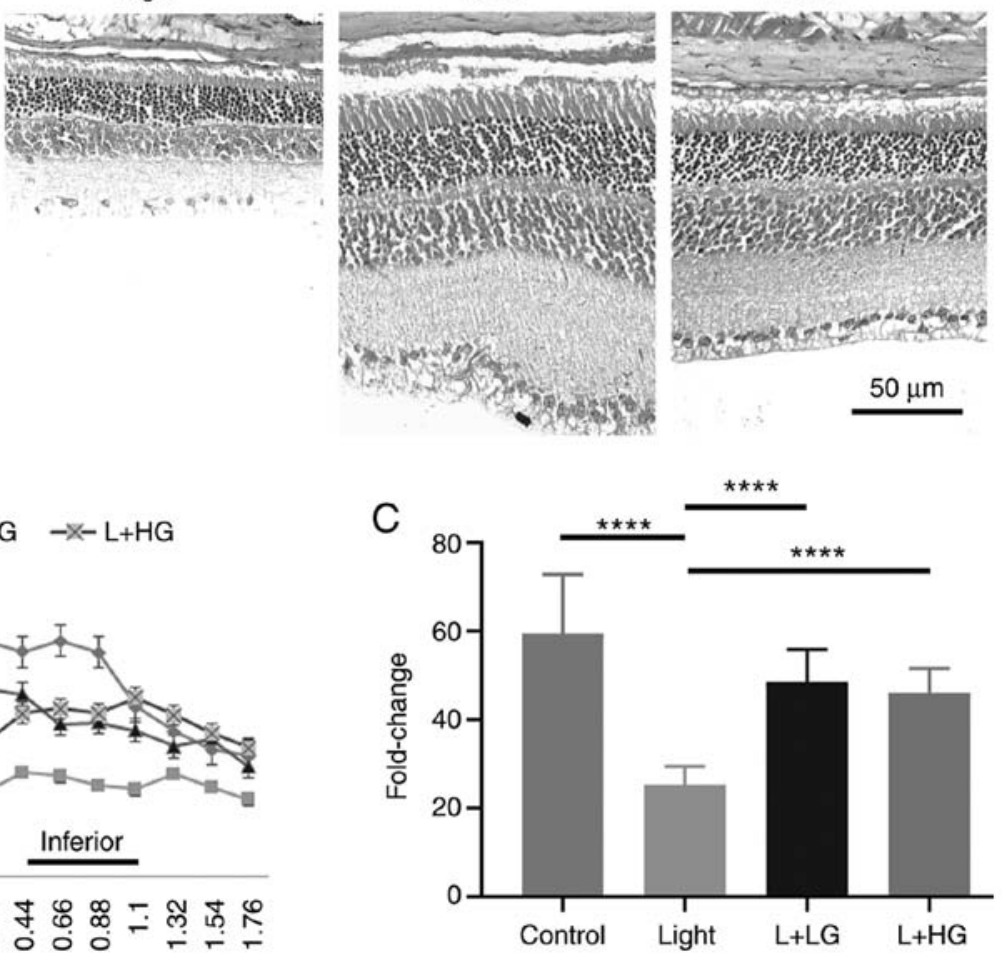

Figure 2. Protective effect of EGb-761 in light-induced photoreceptor degeneration. (A) Effect of EGb-761 on the morphology of the retina in light-damaged mice (magnification, $x 400$ ). (B) Spider graph of ONL thickness in the light-induced retinal degeneration groups. (C) Thickness of the ONL in light-treated mice. The data are presented as the mean $\pm \mathrm{SD}(\mathrm{n}=3 \text { in each group })^{* * * *} \mathrm{P}<0.0001$. EGb-761, L + HG (100 mg/kg); L + LG (50 mg/kg); ONL, outer nuclear layer; RPE, retinal pigment epithelium; OS, IS, INL, inner nuclear layer; GCL, ganglion cell layer.

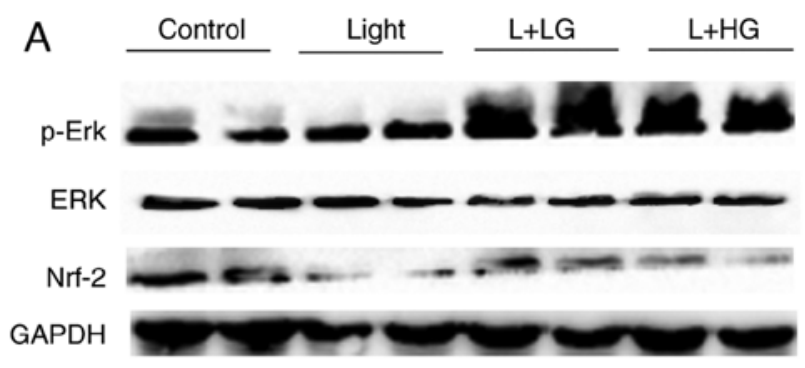

C

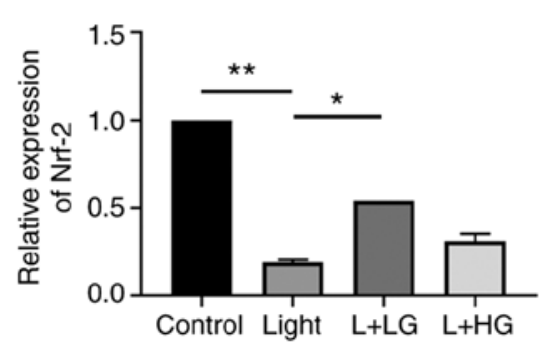

$\mathrm{D}$

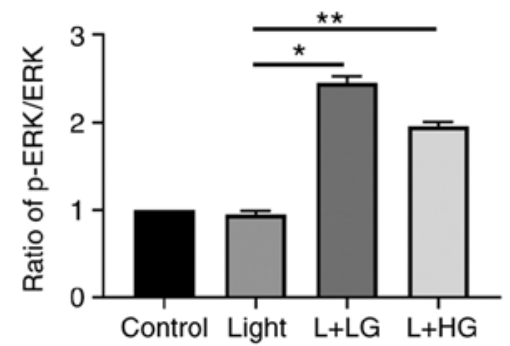

B

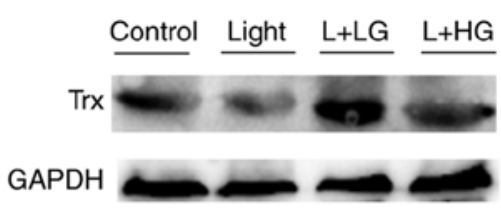

$\mathrm{E}$

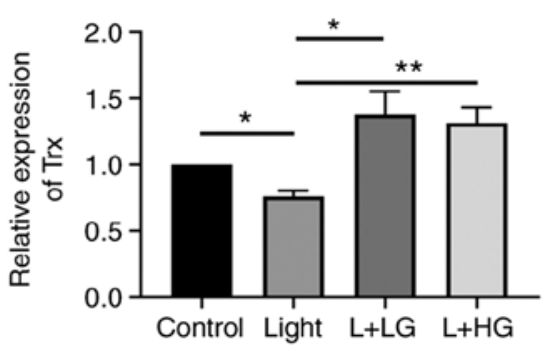

Figure 3. EGb 761 treatment increases the protein levels of Trx, Nrf-2 and p-Erk in light-induced mouse retina degeneration. Protein expression levels of (A) Nrf-2, p-Erk and (B) Trx were detected by western blotting. Semi-quantification of (C) Nrf-2, (D) p-Erk and (E) Trx. The data are presented as the mean $\pm \mathrm{SD}\left(\mathrm{n}=4\right.$ in each group). ${ }^{*} \mathrm{P}<0.05,{ }^{* *} \mathrm{P}<0.01 . \mathrm{L}+\mathrm{HG}(100 \mathrm{mg} / \mathrm{kg})$; L + LG (50 mg/kg); Nrf-2, nuclear factor erythroid 2-related factor 2; p-, phosphorylated; Trx, thioredoxin.

loss in photoreceptor cells, which reduced the ONL thickness of the retina in the light-induced retinal degeneration group compared with the control group (Fig. 2). However, the process could be prevented by pre-treatment with EGb-761 (LG and HG; Fig. 2). These findings suggested that EGb-761 could delay photoreceptor degeneration and improve the visual and 

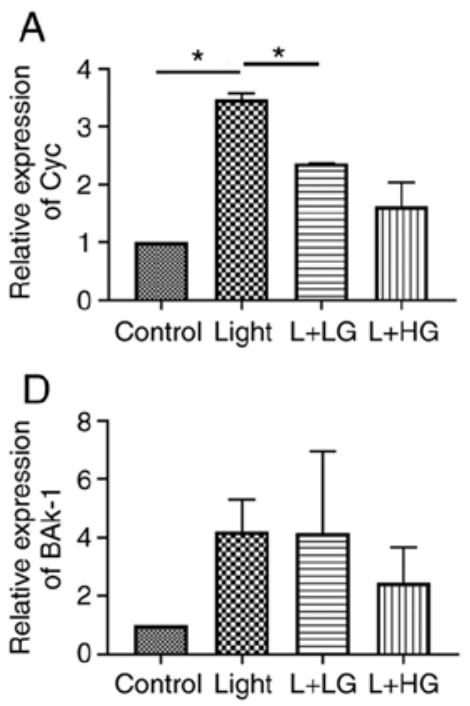

B

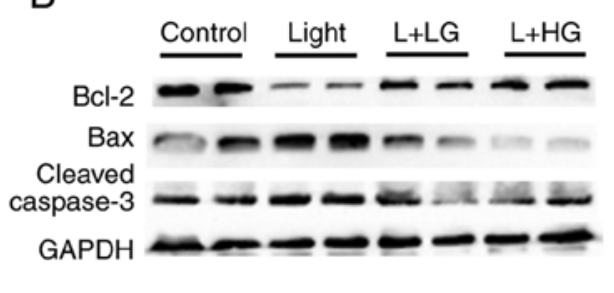

$\mathrm{E}$

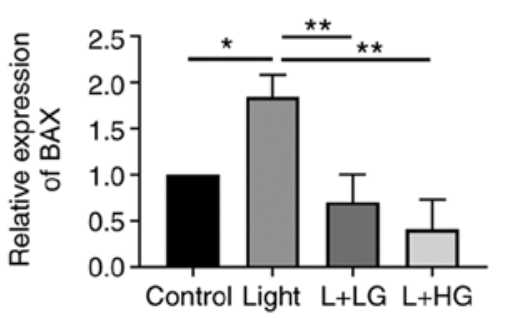

C

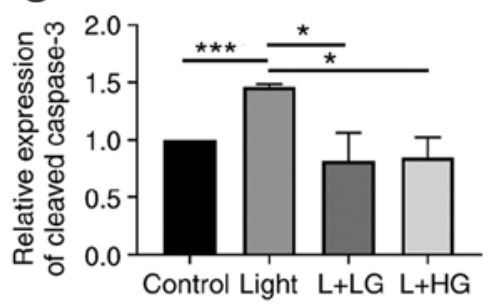

F

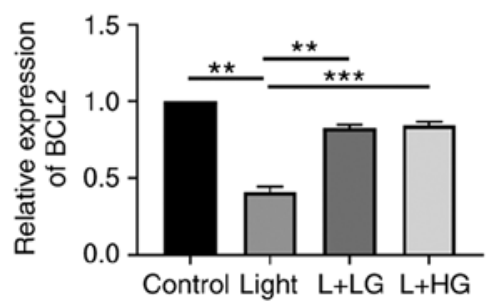

Figure 4. EGb 761 treatment decreases the expression levels of Cyc, Bax, Bak-1 and cleaved caspase-3, and increases the expression levels of Bcl2 in light-induced mouse retina degeneration. The expression levels of (A) Cyc and (B) Bak-1 were detected using reverse transcription-quantitative PCR. (C) Expression levels of cleaved caspase-3, Bax and Bcl2 were detected using western blotting. Semi-quantification of (D) cleaved caspase-3, (E) Bax and (F) Bcl2. The data are presented as the mean $\pm \mathrm{SD}\left(\mathrm{n}=4\right.$ in each group). ${ }^{*} \mathrm{P}<0.05,{ }^{* *} \mathrm{P}<0.01,{ }^{* * * *} \mathrm{P}<0.001$. Bak-1, BCL2 antagonist/killer $1 ; \mathrm{Cyc}, \mathrm{cytochrome} c$; $\mathrm{L}+\mathrm{HG}(100 \mathrm{mg} / \mathrm{kg})$; $\mathrm{L}+\mathrm{LG}(50 \mathrm{mg} / \mathrm{kg})$.

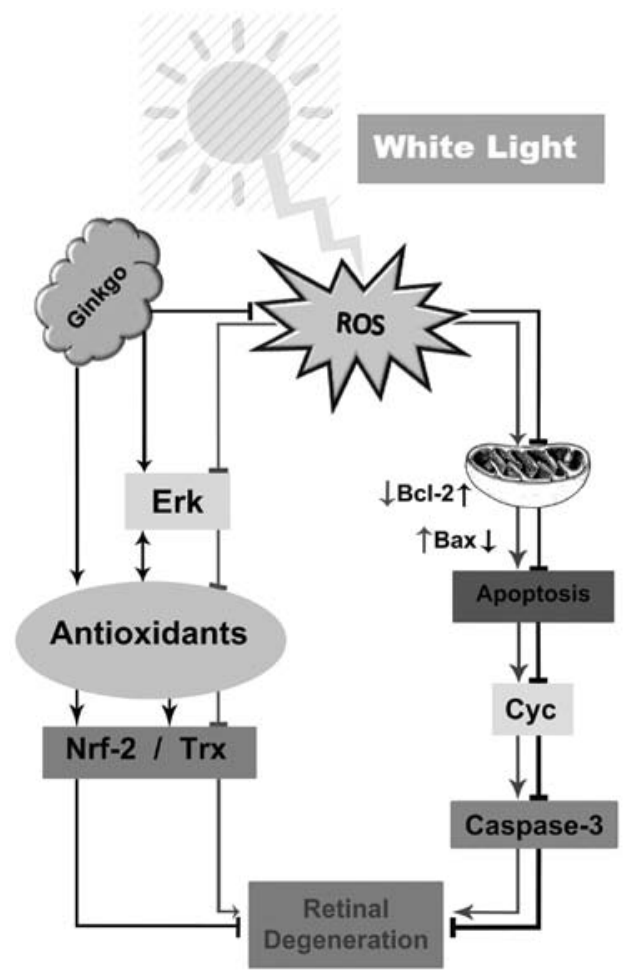

Figure 5. Schematic summary of the protective effects of EGb 761 against light-induced photoreceptor degeneration. Cyc, cytochrome c; Nrf-2, nuclear factor erythroid 2-related factor 2; ROS, reactive oxygen species; Trx, thioredoxin.

morphological function of the retina during light-induced damage.

Effect of EGb-761 treatment on the p-Erk/Nrf-2/Trx signaling pathway in mice exposed to light. The in vivo findings suggested that the protein levels of Nrf-2/Trx were significantly decreased in the light-induced retinal degeneration group compared with the control group. However, pre-treatment with EGb-761 significantly increased the p-Erk/Nrf-2/Trx levels compared with those in the light-induced retinal degeneration group. However, there is no significant difference between that in the Light and $\mathrm{L}+\mathrm{HG}$ groups for $\mathrm{Nrf}-2$ (Fig. 3). These results indicated that treatment with EGb-761 reduced oxidative stress-induced by light damage by activating the p-Erk/Nrf-2/Trx signaling pathway.

EGb-761 treatment decreases the expression levels of proteins of the cleaved caspase-3/Bax/Bak-1/Cyc signaling pathway and increases Bcl-2 expression. In order to investigate the effects of EGb-761 on light-induced photoreceptor degeneration, RT-qPCR and western blotting were performed. Quantitative analysis of mRNA (Fig. 4A and B) indicated that the expression levels of Cyc and Bak-1 were increased in the light-induced retinal degeneration group compared with the control group. However, there is no significance in the difference among the groups for Bak-1. However, the expression levels of Cyc and Bak-1 were decreased following EGb-761 treatment compared with those in the light-induced retinal degeneration group. In addition, the protein expression levels of cleaved caspase-3, Bax and Bcl-2 (Fig. 4C) were detected by western blotting. The expression levels of cleaved caspase-3 and Bax were increased in the light-induced retinal degeneration group compare with those in the control group, which were in turn downregulated following EGb-761 pre-treatment compared with those in the light damage group, (Fig. 4D and E). Furthermore, the expression levels of Bcl-2 were decreased in the light-induced retinal degeneration group, and they were significantly increased following treatment with EGb-761 (Fig. 4F). The results indicated that the expression levels of cleaved caspase-3/Bax/Cyc were significantly increased in the light-induced retinal degeneration group compared with the control group, whereas this effect was inhibited following treatment of the mice with EGb-761. This suggests that the cleaved caspase-3/Bax/Cyc 
pathway is the mechanism through which light induces photoreceptor degeneration and also showed that EGb 761 inhibits the effects of light on retinal degeneration through activating the p-ERK/Nrf2/Trx pathway (Fig. 5).

\section{Discussion}

In the present study, the protective effects of $E G b 761$ were assessed in retinal degeneration. In addition, the molecular mechanisms involved in the regulation of oxidative stress and the cell death signaling pathway induced by white light (5,000 lux) were explored. The results suggested that prolonged exposure to white light led to photoreceptor cell degeneration, characterized by the activation of the corresponding apoptotic signaling pathway. However, little is known regarding the signaling pathway associated with these processes. Treatment of animals with EGb-761 markedly reduced oxidative stress via activation of the Nrf-2/Trx/Erk signaling pathway. By contrast to this effect, treatment of the animals with $E G b 761$ reduced apoptosis via the regulation of the $\mathrm{Bax} / \mathrm{Bcl} 2$ and caspase signaling pathways. Therefore, the observations suggested that $E G b 761$ may serve a crucial role in the clinical treatment of retinal degenerative diseases.

The severity of light-induced retinal impairment is commonly associated with the induction of oxidative stress, which is dependent on the light intensity and exposure time (32). In various studies $(23,29,33)$, the rat/mouse model has been exposed to a variety of white light sources, with different intensities that ranged between 1,000 and 20,000 lux $(6,7,8,12)$. In addition, different irradiation rhythms, different light sources and different spectra have different effects on the retina (3). It has been reported in previous studies, that the decrease of light intensity and alteration in the light constituents can reduce the effect of retinal degeneration $(3,6,34)$. These conditions are known to cause retinal histopathological and functional changes in animals $(6,35)$. Therefore, the present study evaluated the effects of exposure to 5,000 lux of light for $24 \mathrm{~h}$ in a mouse model by focusing on the assessment of different retinal functions. The selected range was based on previous study (6). Furthermore, our research group has used the same range in previous study (3). Therefore, in the present study, this range was selected. In the present study, prolonged exposure to white light triggered a large burst in photoreceptor cell death, which resembled the pathogenesis of retinal degeneration. The results suggested that the induction of morphological and functional changes following white light (5,000 lux) exposure contributed to the development of retinal diseases. The amplitudes of the $a$-wave and b-wave determined the induction of specific disorders associated with retinal function, and the amplitudes were decreased in the light damage group and were accompanied by loss of photoreceptor cells, reducing ONL thickness in the outer segment of the retina. These results were similar to those reported previously (29).

EGb-761 is used for the treatment of different diseases, such as neurodegenerative and retinal disorders (19). Functional and morphological analysis demonstrated that EGb-761 could inhibit light-induced retinal damage. These results support previously reported data (23). However, little is known regarding the molecular mechanism of EGb 761. Therefore, the present study investigated the mechanism by which EGb 761exerted a protective effect against light-induced retinal degeneration. Previous literature reviews have reported that the effect of EGb 761 is dose-dependent $(36,37)$. A dose range of $40-300 \mathrm{mg} / \mathrm{kg}$ has been found to be more effective in the treatment of specific diseases $(38,39)$. The present study demonstrated that treatment with a low dose $(50 \mathrm{mg} / \mathrm{kg})$ of EGb 761 was more effective in preventing retinal degeneration than high-dose treatment $(100 \mathrm{mg} / \mathrm{kg})$.

A previous study has reported that prolonged and intense exposure to light promotes the induction of oxidative stress, which is involved in the pathogenesis of various retinal diseases (40). The Erk/Trx/Nrf-2 signaling pathway serves a pivotal role in redox balance. When the levels of cellular oxidative stress are increased, the p-Erk axis of unfolded protein response mediates nuclear translocation of antioxidant Nrf-2 leading to increased expression of Trx (41), reduced photooxidative stress and retinal degeneration (42). Overall, the p-Erk/Nrf-2/Trx cascade is tightly involved in the regulation of the redox signaling pathway (43). Previous studies have reported that the use of antioxidants is considered the chief regulator of the cellular redox mechanism mediated via the $\mathrm{p}$-Erk/Nrf-2/Trx axis $(3,44)$. This signaling pathway (p-Erk/Nrf-2/Trx) can maintain the biological and physiological function of the retina (45). In the present study, EGb 761 treatment increased the levels of Trx, Nrf-2 and p-Erk in a light damage mouse model. Therefore, the results suggested that EGb 761 acted as an antioxidant that could serve a vital role in retinal degeneration.

The excess levels of ROS and oxidative stress initiate apoptosis or programmed cell death (46), which causes photoreceptor cell death leading to various retinal diseases and blindness (47). The Bak-1/Cyc/Bax/cleaved caspase-3 signaling pathway has attracted considerable attention and is considered the major cause of photoreceptor cell death noted in retinal diseases (47-49). The cell death mechanism is initiated by the translocation of Bax and Bak to the mitochondrial membrane, and Cyc release into the cytosol $(50,51)$. This initiates the activation of cleaved caspase-3, suppressing $\mathrm{Bcl} 2$, and induces morphological and biochemical changes in the retina $(25,26)$. The results of the present study indicated that exposure to light upregulated the levels of Cyc, Bak-1, Bax and cleaved caspase- 3 in the retina, while this process was inhibited following pretreatment of the mice with $\mathrm{EGb}$ 761. This effect could protect photoreceptor cells from apoptosis.

Overall, the data demonstrated that EGb 761 treatment delayed photoreceptor degeneration in light-exposed mice. The mechanism involved activation of the p-Erk/Nrf-2/Trx axis and downregulation of the apoptotic Bax/Bak-1/Cyc/cleaved caspase-3 signaling pathway, which was concomitant with the upregulation of $\mathrm{Bcl} 2$ expression. These results may suggest a putative role of EGb 761 in the treatment of retinal degeneration. The lack of measurements of Cyc release into the cytosol and measurements of mitochondrial membrane potential was a limitation of the present study.

In conclusion, the present study suggested that EGb 761 treatment delayed photoreceptor degeneration induced by light. The underlying mechanism was associated with inhibition of apoptosis via downregulation of the Bax/Cyc/cleaved 
caspase-3 signaling pathway and upregulation of $\mathrm{Bcl} 2$, which led to inhibition of oxidative stress via the activation of the p-Erk/Nrf-2/Trx signaling pathway.

\section{Acknowledgements}

The authors would like to thank Miss Jinjuan Lv, Miss Limin Wei and Miss Jiao Li, Department of Histology and Embryology, Dalian Medical University (Dalian, China) for their technical assistance during the experiments.

\section{Funding}

The present study was supported from the National Natural Science Foundation of China (grant no. 31371218) and the Basic Scientific Research Projects of the Liaoning Provincial Education Department (grant no. LQ2017005). This work was also supported by the Natural Science Foundation of Liaoning Province (grant no. 2020-BS-189). The Liaoning Provincial Program supported this work for the Top Discipline of Basic Medical Sciences.

\section{Availability of data and materials}

The datasets used and/or analyzed during the current study are available from the corresponding author on reasonable request.

\section{Authors' contributions}

MC and $\mathrm{CZ}$ designed the study, carried out experiments, analyzed the data, and wrote and revised the manuscript. SS contributed to the experiments and revised the manuscript. $\mathrm{XR}$ and LK designed, supervised and provided funding for the experiments. XR and LK also checked the analyzed data and revised the manuscript critically for important intellectual content revised. XR and LK are responsible for confirming the authenticity of the raw data. All authors read and approved the final manuscript.

\section{Ethics approval and consent to participate}

All procedures were approved by the Institutional Animal Care and Use Committee of the Dalian Medical University (Dalian, China).

\section{Patient consent for publication}

Not applicable.

\section{Competing interests}

The authors declare that they have no competing interests.

\section{References}

1. Nash TR, Chow ES, Law AD, Fu SD, Fuszara E, Bilska A Bebas P, Kretzschmar D and Giebultowicz JM: Daily blue-light exposure shortens lifespan and causes brain neurodegeneration in Drosophila. NPJ Aging Mech Dis 5: 8, 2019.

2. Contín M, Benedetto M, Quinteros-Quintana M and Guido M: Light pollution: The possible consequences of excessive illumination on retina. Eye (Lond) 30: 255-263, 2016.
3. Kong L, Liu B, Zhang C, Wang B, Wang H, Song X, Yang Y Ren $X$, Yin L, Kong $H$ and Ma $H$ : The therapeutic potential of sulforaphane on light-induced photoreceptor degeneration through antiapoptosis and antioxidant protection. Neurochem Int 100: 52-61, 2016.

4. Hu Z, Zhang Y, Wang J, Mao P, Lv X, Yuan S, Huang Z, Ding Y, Xie P and Liu Q: Knockout of Ccr2 alleviates photoreceptor cell death in rodent retina exposed to chronic blue light. Cell Death Dis 7: e2468, 2016.

5. Murakami Y, Notomi S, Hisatomi T, Nakazawa T, Ishibashi $\mathrm{T}$, Miller JW and Vavvas DG: Photoreceptor cell death and rescue in retinal detachment and degenerations. Prog Retin Eye Res 37: 114-140, 2013.

6. Schäfer N, Grosche A, Schmitt SI, Braunger BM and Pauly D: Complement components showed a time-dependent local expression pattern in constant and acute white light-induced photoreceptor damage. Front Mol Neurosci 10: 197, 2017.

7. Maeda A, Palczewska G, Golczak M, Kohno H, Dong Z, Maeda T and Palczewski K: Two-photon microscopy reveals early rod photoreceptor cell damage in light-exposed mutant mice. Proc Natl Acad Sci USA 111: E1428-E1437, 2014.

8. Bian M, Du X, Cui J, Wang P, Wang W, Zhu W, Zhang T and Chen Y: Celastrol protects mouse retinas from bright light-induced degeneration through inhibition of oxidative stress and inflammation. J Neuroinflammation 13: 50, 2016.

9. Masuda T, Shimazawa M and Hara H: Retinal diseases associated with oxidative stress and the effects of a free radical scavenger (Edaravone). Oxid Med Cell Longev 2017: 9208489, 2017.

10. Smolková K, Mikó E, Kovács T, Leguina-Ruzzi A, Sipos A and Bai P: Nuclear factor erythroid 2-related factor 2 in regulating cancer metabolism. Antioxid Redox Signal 33: 966-997, 2020.

11. Canning P, Sorrell FJ and Bullock AN: Structural basis of Keap1 interactions with Nrf-2. Free Radic Biol Med 88: 101-107, 2015.

12. Tanito M, Kwon YW, Kondo N, Bai J, Masutani H, Nakamura H, Fujii J, Ohira A and Yodoi J: Cytoprotective effects of geranylgeranylacetone against retinal photooxidative damage. J Neurosci 25: 2396-2404, 2005.

13. Lv J, Bao S, Liu T, Wei L, Wang D, Ye W, Wang N, Song S, Li J, Chudhary M, et al: Sulforaphane delays diabetes-induced retinal photoreceptor cell degeneration. Cell Tissue Res 382: 477-486, 2020.

14. Nishinaka Y, Masutani H, Nakamura H and Yodoi J: Regulatory roles of thioredoxin in oxidative stress-induced cellular responses. Redox Rep 6: 289-295, 2001.

15. Cebula M, Schmidt EE and Arnér ESJ: TrxR1 as a potent regulator of the Nrf2-Keap1 response system. ARS 23: 823-853, 2015.

16. Ren X, Sun H, Zhang C, Li C, Wang J, Shen J, Yu D and Kong L: Protective function of pyridoxamine on retinal photoreceptor cells via activation of the p-Erk1/2/Nrf-2/Trx/ASK1 signalling pathway in diabetic mice. Mol Med Rep 14: 420-424, 2016.

17. Isah T: Rethinking Ginkgo biloba L.: Medicinal uses and conservation. Pharmacogn Rev 9: 140-148, 2015.

18. Zuo W, Yan F, Zhang B, Li J and Mei D: Advances in the studies of Ginkgo biloba leaves extract on aging-related diseases. Aging Dis 8: 812-826, 2017.

19. Zaghlool SS, Hanaf LK, Afifi NM and Ibrahim ER: Histological and immunohistochemical study on the protective effect of Ginkgo biloba extract against glutamate-induced neurotoxicity in male albino rat retinal cells. Egypt J Histol 35: 176-188, 2012.

20. Chen XJ, Ren SM, Dong JZ, Qiu CG, Chen YW and Tao HL: Ginkgo biloba extract-761 protects myocardium by regulating Akt/Nrf-2 signal pathway. Drug Des Devel Ther 13: 647-655, 2019.

21. Liu SQ, Xu CY, Qin MB, Tan L, Zhuge CF, Mao YB, Lai MY and Huang JA: Ginkgo biloba extract enhances chemotherapy sensitivity and reverses chemoresistance through suppression of the KSR1-mediated ERK1/2 pathway in gastric cancer cells. Oncol Rep 33: 2871-2882, 2015.

22. Czauderna C, Palestino-Dominguez M, Castven D, Becker D, Zanon-Rodriguez L, Hajduk J, Mahn FL, Herr M, Strand D, Strand S, et al: Ginkgo biloba induces different gene expression signatures and oncogenic pathways in malignant and non-malignant cells of the liver. PLoS One 13: e0209067, 2018.

23. Ranchon I, Gorrand JM, Cluzel J, Droy-Lefaix MT and Doly M: Functional protection of photoreceptors from light-induced damage by dimethylthiourea and Ginkgo biloba extract. Invest Ophth Vis 40: 1191-1199, 1999.

24. Finucane DM, Bossy-Wetzel E, Waterhouse NJ, Cotter TG and Green DR: Bax-induced caspase activation and apoptosis via cytochromec release from mitochondria is inhibitable by Bcl-xL. J Biol Chem 274: 2225-2233, 1999. 
25. Arango-Gonzalez B, Trifunović D, Sahaboglu A, Kranz K, Michalakis S, Farinelli P, Koch S, Koch F, Cottet S, Janssen-Bienhold U, et al: Identification of a common non-apoptotic cell death mechanism in hereditary retinal degeneration. PLoS One 9: e112142, 2014.

26. Ali D, Tripathi A, Al Ali H, Shahi Y, Mishra KK, Alarifi S, Alkahtane AA and Manohardas S: ROS-dependent Bax/Bcl2 and caspase 3 pathway-mediated apoptosis induced by zineb in human keratinocyte cells. Onco Targets Ther 11: 489, 2018

27. Wang A, Yang Q, Li Q, Wang X, Hao S, Wang J and Ren M: Ginkgo Biloba L. extract reduces $\mathrm{H} 2 \mathrm{O} 2$-induced bone marrow mesenchymal stem cells cytotoxicity by regulating mitogen-activated protein kinase (MAPK) signaling pathways and oxidative stress. Med Sci Mon Int Med J Exp Clin Res 24: 3159-3167, 2018

28. Wang Y, Lv J, Cheng Y, Du J, Chen D, Li C and Zhang J: Apoptosis induced by Ginkgo biloba (EGb761) in melanoma cells is Mcl-1-dependent. PLoS One 10: e0124812, 2015.

29. Tang L, Bao S, Du Y, Jiang Z, Wuliji A, Ren X, Zhang C, Chu H, Kong L and Ma H: Antioxidant effects of Lycium barbarum polysaccharides on photoreceptor degeneration in the light-exposed mouse retina. Biomed Pharmacother 103: 829-837, 2018.

30. Liu J, Wei L, Wang Z, Song S, Lin Z, Zhu J, Ren X and Kong L: Protective effect of Liraglutide on diabetic retinal neurodegeneration via inhibiting oxidative stress and endoplasmic reticulum stress. Neurochem Int: 104624, 2019.

31. Livak KJ and Schmittgen TD: Analysis of relative gene expression data using real-time quantitative PCR and the 2(-Delta Delta $\mathrm{C}(\mathrm{T})$ ) method. Methods 25: 402-408, 2001.

32. Lin CW, Yang CM and Yang CH: Effects of the emitted light spectrum of liquid crystal displays on light-induced retinal photoreceptor cell damage. Int J Mol Sci 20: 2318, 2019.

33. Randazzo J, Zhang Z, Hoff M, Kawada H, Sachs A, Yuan Y, Haider $\mathrm{N}$ and Kador P: Orally active multi-functional antioxidants are neuroprotective in a rat model of light-induced retinal damage. PLoS One 6: e21926, 2011.

34. Chu-Tan JA, Rutar M, Saxena K, Wu Y, Howitt L, Valter K, Provis J and Natoli R: Efficacy of $670 \mathrm{~nm}$ light therapy to protect against photoreceptor cell death is dependent on the severity of damage. Int J Photoenergy 2016, 2016.

35. Iliescu DA, Ciubotaru A, Ghiță MA, Dumitru A and Zăgrean L: Effect of sevoflurane preconditioning on light-induced retinal damage in diabetic rats. Rom J Ophthalmol 62: 24-33, 2018.

36. Ma K, Xu L, Zhan H, Zhang S, Pu M and Jonas J: Dosage dependence of the effect of Ginkgo biloba on the rat retinal ganglion cell survival after optic nerve crush. Eye (Lond) 23: 1598-1604 2009.

37. Biddlestone L, Corbett AD and Dolan S: Oral administration of Ginkgo biloba extract, EGb-761 inhibits thermal hyperalgesia in rodent models of inflammatory and post-surgical pain. $\mathrm{Br}$ J Pharmacol 151: 285-291, 2007.
38. Cheng D, Liang B and Li Y: Antihyperglycemic effect of Ginkgo biloba extract in streptozotocin-induced diabetes in rats. Biomed Res Int 2013: 162724, 2012.

39. Okumus S, Taysi S, Orkmez M, Saricicek E, Demir E, Adli M and Al B: The effects of oral Ginkgo biloba supplementation on radiation-induced oxidative injury in the lens of rat. Pharmacogn Mag 7: 141-145, 2011.

40. Kernt M, Walch A, Neubauer AS, Hirneiss C, Haritoglou C, Ulbig MW and Kampik A: Filtering blue light reduces light-induced oxidative stress, senescence and accumulation of extracellular matrix proteins in human retinal pigment epithelium cells. Clin Exp Ophthalmol 40: e87-e97, 2012.

41. Aydin Y, Chedid M, Chava S, Williams DD, Liu S, Hagedorn CH, Sumitran-Holgersson S, Reiss K, Moroz K, Lu H, et al: Activation of PERK-Nrf-2 oncogenic signaling promotes Mdm2-mediated $\mathrm{Rb}$ degradation in persistently infected HCV culture. Sci Rep 7: 9223, 2017.

42. Munemasa Y, Ahn J, Kwong J, Caprioli J and Piri N: Redox proteins thioredoxin 1 and thioredoxin 2 support retinal ganglion cell survival in experimental glaucoma. Gene Ther 16: 17-25, 2009.

43. Kong L, Tanito M, Huang Z, Li F, Zhou X, Zaharia A, Yodoi J, McGinnis JF and Cao W: Delay of photoreceptor degeneration in tubby mouse by sulforaphane. J Neurochem 101: 1041-1052, 2007.

44. Wu L, Gao L, Cao Y, Chen F, Sun T and Liu Y: Analysis of the protective mechanism of liraglutide on retinopathy based on diabetic mouse model. Saudi J Biol Sci 26: 2096-2101, 2019.

45. Ma Q: Role of Nrf-2 in oxidative stress and toxicity. Annu Rev Pharmacol Toxicol 53: 401-426, 2013.

46. Ryter SW, Kim HP, Hoetzel A, Park JW, Nakahira K, Wang X and Choi AM: Mechanisms of cell death in oxidative stress. Antioxid Redox Signal 9: 49-89, 2007.

47. Lo AC, Woo TT, Wong RL and Wong D: Apoptosis and other cell death mechanisms after retinal detachment: Implications for photoreceptor rescue. Ophthalmologica 226 (Suppl 1): S10-S17, 2011.

48. Peter ME, Heufelder AE and Hengartner MO: Advances in apoptosis research. Proc Natl Acad Sci USA 94: 12736-12737, 1997.

49. D'amelio M, Cavallucci V and Cecconi F: Neuronal caspase-3 signaling: Not only cell death. Cell Death Differ 17: 1104-1114, 2010.

50. Adams JM and Cory S: Bcl-2-regulated apoptosis: Mechanism and therapeutic potential. Curr Opin Immunol 19: 488-496, 2007.

51. Gogada R, Prabhu V, Amadori M, Scott R, Hashmi S and Chandra D: Resveratrol induces p53-independent, X-linked inhibitor of apoptosis protein (XIAP)-mediated Bax protein oligomerization on mitochondria to initiate cytochrome c release and caspase activation. J Biol Chem 286: 28749-28760, 2011. 